The other primary outcome to assess efficacy was defined as the rate of participants not receiving the allocated treatment plus the rate of participants who discontinued the allocated treatment. Even when accepting that patients not even starting treatment were included in a measure of treatment efficacy, it seems problematic to ascribe differences in this criterion to the efficacy of transference-focused psychotherapy without excluding accumulative effects of alternative explanations. The higher rate of non-starters among patients randomised to community therapists (the control condition) compared with those randomised to transference-focused psychotherapy $(25 \% \quad v$. $13 \%)$ and the substantially higher rate of patients stopping treatment in the control group within the first month (Fig. 2 of the paper) might reflect a general preference of participants for transference-focused psychotherapy rather than its superior efficacy. Furthermore, the authors have not mentioned that this criterion combining non-starters and 'drop-outs' as primary outcome of efficacy was introduced post hoc (for post-hoc changes in the definition of primary outcome criteria see http:// clinicaltrials.gov/archive/NCT00714311). Without addressing this issue, the statistical implications of this proceeding are difficult to evaluate.

As for the secondary outcome measures, the picture seems to be mixed. Some of the LOCF analyses indicated lower scores after transference-focused psychotherapy (e.g. number of borderline criteria, level of personality organisation). Other scores (e.g. general psychopathology, depression) were numerically higher after transference-focused psychotherapy and did not improve significantly more under it $(P=0.92$ and $P=0.85$ for general psychopathology and depression respectively).

Recapitulating, it seems that the claimed efficacy of transference-focused psychotherapy does not follow from the primary outcome criteria. Accordingly, further research seems necessary to establish the efficacy of this therapy in the treatment of borderline personality disorder.

1 Doering S, Hörz S, Rentrop M, Fischer-Kern M, Schuster P, Benecke C et al. Transference-focused psychotherapy $v$. treatment by community psychotherapists for borderline personality disorder: randomised controlled trial. Br J Psychiatry 2010; 196: 389-95.

Nikolaus Kleindienst, Department of Psychosomatic Medicine, Central Institute of Mental Health, J5, D-68159 Mannheim, Germany, email: Nikolaus.Kleindienst@ zi-mannheim.de; Bertram Krumm, Department for Biostatistics, Martin Bohus Zi-mannheim.de; Bertram Krumm, Department for Biostatistics, Martin Bohu
Department of Psychosomatic Medicine, Central Institute of Mental Health, Mannheim, Germany

doi: 10.1192/bjp.198.2.156b

Author's reply: Kleindienst and colleagues argue that our interpretation of the treatment outcome with regard to suicide attempts might lead to misunderstandings. They are right that a $\chi^{2}$-test comparing the absolute number of suicide attempters in both groups, transference-focused psychotherapy and treatment by experienced community psychotherapists, is not significant. However, this test does not seem appropriate in the present context, since the baseline, that is the number of suicide attempters during the year before treatment, was not equal in both groups (18 in the transference-focused psychotherapy group $v .12$ in the community psychotherapists group). Thus, a statistical approach had to be employed that controls for baseline data. Since no $\chi^{2}$-test exists that controls for baseline values, we defined change scores that allow for baseline control within a MannWhitney U-test. This test generated the $P=0.009$ that, in our opinion, depicts the real changes in suicide attempters in both groups. A between-group effect size of 0.55 for the time $\times$ group interaction in suicide attempts was calculated from the $\chi^{2}$-statistics of the change scores $\left(\chi^{2}=7.126\right.$, d.f. $\left.=2, P<0.028\right)$. Table DS2 of our paper only reports within-group effect sizes; between-group effect sizes were not calculated.

The issue of treatment drop-out is a limitation of this study, which has been thoroughly controlled for and discussed in our paper. After the decision to use treatment drop-out as a primary outcome criterion, we preferred to keep strictly to the intent-totreat algorithm that demands every randomised patient to be part of the outcome analysis. Nevertheless, to address the understandable criticism raised by Kleindienst and colleagues, we repeated the drop-out analysis after excluding from it patients who did not begin therapy after randomisation. This analysis still revealed a significantly lower number dropping out of the transference-focused psychotherapy group $\left(15\right.$ v. $23 ; \chi^{2}=5.750$, d.f. $=1, P=0.016)$.

The changes in the primary outcome criteria had been made following the impression of an ongoing discussion in the literature addressing the adequacy of DSM-IV diagnostic criteria as outcome criteria in treatment studies. ${ }^{1,2}$ Since our initial outcome criteria 'number of DSM-IV borderline criteria' and 'GAF score' revealed an even stronger superiority of transference-focused psychotherapy, we did not report this post-hoc change, because a bias in our decision was not suspected.

We thank Kleindienst and colleagues for their criticism and the Editor for giving us the opportunity to clarify important issues regarding our study. We hope that our comments will eliminate doubts concerning the fact that our study documents the efficacy of transference-focused psychotherapy for the treatment of borderline personality disorder.

1 Skodol AE, Pagano ME, Bender DS, Shea MT, Gunderson JG, Yen S, et al. Stability of functional impairment in patients with schizotypal, borderline, avoidant, or obsessive-compulsive personality disorder over two years. Psychol Med 2005; 35: 443-51.

2 Zanarini MC, Frankenburg FR, Reich DB, Silk KR, Hudson Jl, McSweeney LB. The subsyndromal phenomenology of borderline personality disorder: a 10-year follow-up study. Am J Psychiatry 2007; 164: 929-35.

Stephan Doering, Department of Psychoanalysis and Psychotherapy, Medica University of Vienna, Austria, email: Stephan.Doering@ukmuenster.de

doi: 10.1192/bjp.198.2.157

\section{Ziprasidone and the relative risk of diabetes}

Kessing et al $^{1}$ describe the risks of diabetes in clinical practice from a large-cohort, observational study of Danish patients requiring antipsychotics. We believe that the relative risks of subsequent incident diabetes that they report for individual antipsychotics are at odds with established literature. The preponderance of evidence has demonstrated that ziprasidone has limited effect on metabolic indices associated with the development of diabetes. We present some of that evidence below.

In the CATIE study of 1493 patients with schizophrenia receiving olanzapine, quetiapine, risperidone, ziprasidone or perphenazine for up to 18 months, ziprasidone was the only drug associated with improvement in glycosylated haemoglobin, total cholesterol and triglycerides. Meyer and colleagues ${ }^{2}$ reported that, in the CATIE trial, the prevalence of metabolic syndrome increased for olanzapine (from $34.8 \%$ to $43.9 \%$ ) but decreased for ziprasidone (from $37.7 \%$ to $29.9 \%$ ), and that the comparison between ziprasidone and olanzapine was statistically significant $(P=0.001)$.

In the EUFEST study of 498 patients with first-episode schizophrenia assigned to haloperidol, amisulpride, olanzapine, 
quetiapine or ziprasidone for 12 months, weight change was greatest on olanzapine and among the lowest on ziprasidone. ${ }^{3}$ Ziprasidone was not associated with untoward effects on any other metabolic risk factors.

A pooled analysis in 2009 of over 100 Pfizer-sponsored randomised controlled trials found no significant differences between 1605 individuals given ziprasidone and 677 given placebo in total cholesterol, fasting glucose or fasting triglycerides (details available on request).

Yood et $a{ }^{4}{ }^{4}$ in a 55287 -member inception cohort of antipsychotic users, found 357 cases of newly treated diabetes. Ziprasidone was among the group of agents with the lowest risk of diabetes. Patients exposed to olanzapine and clozapine had an increased risk of the illness.

A consensus statement on antipsychotic drugs and obesity published by the American Diabetes Association et al in 2004 concluded that increased risks of obesity, dyslipidaemia and diabetes are most associated with clozapine and olanzapine; little or no significant weight gain, diabetes and dyslipidaemia was associated with aripiprazole and ziprasidone, although it should be noted that these agents had not yet been used extensively at that time. $^{5}$ Further, the panel suggested switching patients who develop worsening glycaemia or dyslipidaemia to a secondgeneration antipsychotic not associated with significant weight gain or diabetes (i.e. ziprasidone or aripiprazole). Standards of practice that promote agents with lower metabolic risks may be a confounding factor in naturalistic studies.

Kessing et al acknowledge that individuals at higher risk of diabetes because of a personal history of obesity or inactivity, a family history of diabetes or other risk factors may have been prescribed agents perceived to confer a lower risk of diabetes'. This channelling bias affects the generalisability of their results.

They report a low risk of diabetes for aripiprazole, but the drug did not becoming commercially available in Denmark until 2004 , only 1.5 years before the end of this 10 -year study. A small number of patients were exposed for a limited period of time, making the direct comparison with ziprasidone not meaningful.

With regard to Table 3 , given the widely differing times of drug exposure and the ultimate position of any individual agent within a single patient's treatment regimen, conclusions about the risk of an individual agent $v$. a drug class may be inappropriate based on this study design.

We are concerned about how clinicians will interpret Kessing et al's findings for ziprasidone, as the results stand in contrast to the relative risks for diabetes reported in the established literature.

1 Kessing LV, Thomsen AF, Mogensen UB, Andersen PK. Treatment with antipsychotics and the risk of diabetes in clinical practice. Br J Psychiatry 2010; 197: 266-71.

2 Meyer JM, Davis VG, Goff DC, McEvoy JP, Nasrallah HA, Davis SM, et al. Change in metabolic syndrome parameters with antipsychotic treatment in the CATIE Schizophrenia Trial: prospective data from phase 1. Schizophr Res 2008; 101: 273-86.

3 Kahn R, Fleischhacker WW, Karayal O, Siu C, Pappadopulos E, EUFEST Study Group (2009) EUFEST: the effects of first and second generation antipsychotics on metabolic and cardiovascular risk factors. In American Psychiatric Association 162nd Annual Meeting, 2009 New Research Abstracts, Abstract NR1-043. APA, 2009

4 Yood MU, DeLorenze G, Quesenberry, CP Jr, Oliveria SA, Tsai AL, Willey VJ, et al. The incidence of diabetes in atypical antipsychotic users according to agent - results from a multisite epidemiologic study. Pharmacoepidemiol Drug Saf 2009; 18: 791-9.

5 American Diabetes Association, American Psychiatric Association, American Association of Clinical Endocrinologists, North American Association for the Study of Obesity. Consensus Development Conference on Antipsychotic Drugs and Obesity and Diabetes (consensus statement). Diabetes Care 2004; 27: 596-601.

\section{Declaration of interest}

D.V., D.K. and O.N.K are employed by Pfizer Inc.

Douglas Vanderburg, Pfizer Inc., 235 East 42nd Street, New York, NY 10017 USA, email: Douglas.Vanderburg@pfizer.com; Denis Keohane, Onur N. Karayal, Elizabeth Pappadopulos, Pfizer Inc., New York, New York, USA

doi: 10.1192/bjp.198.2.157a

Authors' reply: We thank Dr Vanderburg and colleagues for their comments on our paper. We used observational, non-randomised, routinely collected data to describe the rate of incident diabetes among patients treated with antipsychotics in clinical practice. These data reflect the way antipsychotics are handled by individual clinicians on the basis of their knowledge of effects and side-effects for the specific patient. Findings from analyses of our data cannot be used to infer causality between individual antipsychotics and diabetes and may be at odds with findings from randomised trials and other studies aimed at testing specific hypotheses. Our results on the individual antipsychotics describe the prevalence of diabetes among patients for whom the clinician decided to prescribe a given antipsychotic.

\section{Declaration of interest}

L.V.K. has been a consultant for Bristol-Myers Squibb, Eli Lilly, Lundbeck, AstraZenica, Pfizer, Wyeth, Servier and Janssen-Cilag.

Lars Vedel Kessing, Department of Psychiatry, Rigshospitalet, University Hospital of Copenhagen, 2100 Copenhagen, Denmark, email: Lars.Vedel.Kessing@regionh.dk; Per Kragh Andersen, Department of Biostatistics, University of Copenhagen, Copenhagen, Denmark

doi: 10.1192/bjp.198.2.158 